\title{
Development of Interaction Concepts for Touchless Human-Computer Interaction with Geographic Information Systems
}

\author{
Ronald Meyer ${ }^{1}$, Jennifer Bützler ${ }^{1}$, \\ Jeronimo Dzaack ${ }^{2}$, and Christopher M. Schlick ${ }^{1}$ \\ ${ }^{1}$ Institute of Industrial Engineering and Ergonomics, RWTH Aachen University \\ $\{r$.meyer,j.buetzler, c.schlick\}@iaw.rwth-aachen.de \\ http://www.iaw.rwth-aachen.de \\ 2 ATLAS ELEKTRONIK GmbH, Sebaldsbrücker Heerstr. 235, \\ D-28309 Bremen, Germany \\ jeronimo.dzaack@atlas-elektronik.com \\ http://www.atlas-elektronik.com
}

\begin{abstract}
Interaction concepts in 3D GIS are yet limited to 2D input methods like mouse and keyboard. This work describes elaboration of a concept of touchless interaction for a prototype that aims to be used in maritime GIS applications. Experts from the maritime field have been interviewed to construct a rigorous scenario settled in the maritime field. Besides the planning and conversion of a stereoscopic GIS prototype a touchless interaction concept for stereoscopic environments under consideration of three different hand models is developed and presented. Implementation of these different hand models is planned for future evaluation.
\end{abstract}

\section{Introduction}

Geographic information systems (GIS) provide access to geospatial and context situated data for a wide area of applications and target a broad audience of users from novices to professionals in different areas. Traditional GIS applications are visualized 2D with planar top-view. Advances in computer graphics technology allow visualization of $3 \mathrm{D}$ scenes with increasing complexity in real-time. The visualization of GIS benefits from this development since spatial data can now be visualized in 3D virtual space by using modern graphics hardware. Virtual globes like Google Earth 1 or NASA World Wind 2 are versatile tools to exemplify the power of representing spatial relationships in a three-dimensionally rendered environment but both lack of an interaction method that naturally maps on their spatial dimensions. Interaction in three dimensions may be more direct and immersive since there is a shorter cognitive distance between a user's action and the system's feedback through immersion and presence in the virtual world. The feeling of presence, where the physical environment of the user is mentally

${ }^{1}$ http://earth.google.de

${ }^{2}$ http://worldwind.arc.nasa.gov/

M. Kurosu (Ed.): Human-Computer Interaction, Part II, HCII 2014, LNCS 8511, pp. 589-599, 2014. (C) Springer International Publishing Switzerland 2014 
replaced with the virtual one, induces immersion into the virtual environment which facilitates interaction using natural tasks. This allows users to mentally build up complex mental models of e.g. how an interaction concept works 2 .

Since publication of gesture-based game controllers like the Nintendo Wil 1 or the Microsoft Kinect 2 which intend users to interact via body motion or gestures a beginning dissolution of the WIMP (Windows, Icons, Mouse, Pointers) paradigm can be observed also in the non-entertainment area when profiling relevant contributions in the field of human-computer interaction of the recent years. Adaption of technology originating from the entertainment industry has become a famous phenomenon as convenient availability of efficient hardware with stable system abilities is available at low prices through mass market conditions. These new interaction technologies capturing hand and body motion in three dimensions facilitate a reassessment of spatial interaction in virtual globes and 3D GIS.

New devices allow new interaction offering six degrees of freedom (DOF) with millimeter or sub-millimeter accuracy in visual tracking of human body up to finger limbs [10] 17, e.g. the Microsoft Kinect or the Leap Motion controller 3 . These devices' abilities are worth to be investigated in respect to their input precision and reliability in context of a multimodal input for maritime GIS applications since previous research of 3D GIS in maritime context are more focussed on visualization and less on input modalities. Yu et al. see no availability of a true 3D GIS on the current market [18] which is due to several impediments, including deficiencies in data structuring and manipulation of various types of objects, 3D data analysis and large volumes of data.

Information processing in maritime navigation is generally defined through interaction with digital sea maps and virtual object manipulation for navigational purposes. Maritime vehicles and onshore maritime control rooms are likewise equipped with corresponding systems. Maritime safety greatly relies on these systems which must provide availability and accessibility of real-time information through human-computer interactivity. Best possible representation and interaction with data and information allows a high contextual awareness for system users and enhance decision-making processes in time-critical situations.

In this research a concept for touchless human-computer interaction in $3 \mathrm{D}$ GIS is developed putting it's focus on applications in maritime context, i.e. use case scenarios for maritime applications are investigated through interviews with experts on the maritime field. On that basis an interaction concept is developed including a hardware component-off-the-shelf prototype which consists of depth tracking devices like the Microsoft Kinect and the Leap Motion controller and a stereoscopic display which are operated through the open source GIS NASA World Wind in a stereoscopic environment. The prototype forms the basis for future evaluation of different touchless input methods for a maritime 3D GIS.

\footnotetext{
${ }^{1}$ http://www.nintendo.com/wii

2 http://www.microsoft.com/en-us/kinectforwindows/

3 https://www. leapmotion.com/
} 


\section{State of the Art}

Two-dimensionally visualized GIS are prevalent on the commercial market. Mouse and keyboard are adequate input devices as the interaction on 2D maps can easily be achieved through the two degrees of freedom offered by mouse input. With visualizing spatial relations in three dimensions these input devices do not further comply the requirements of interaction and therefore become obsolete.

Modern multimodal input devices as the aforementioned Microsoft Kinect and Leap Motion controller allow a more direct input on spatial data since they provide $3 \mathrm{D}$ output data.

Recent research in the field of multimodal input in 2D and 3D GIS documents the identification of the existing problem, anyway solutions are still in a state of research [18. Rauschert et al. see a necessity of a paradigm shift in the usage of GIS from classical WIMP interaction to multimodal input. Most functionality in GIS is subjected to expert users since special functions can only be accessed via repetitive menu and wizard tool usage [14. Their research reports on a $2 \mathrm{D}$ GIS in a large screen environment that can be operated by spoken commands or free-hand gestural input such as pointing or outlining areas of interest. Fuhrman et al. conducted a user study where a 2D GIS was equipped with multimodal input modalities to rate basic user performance and to generally evaluate user acceptance [5]. The study was conducted under participation of ten voluntary geography graduate students having no or a maximum of three years experience with GIS. The prototype provided functions like data querying, navigating and drawing 2D primitives like points, circles, lines and free-hand drawing. During the study participants were asked to perform tasks like finding a certain map extract, load a data set via speech command or place a marker on the 2D map. The results show that participants with GIS experience had no noticeable advantage in completing tasks over participants with no GIS experience. The participants stated that the speech-based dialogue and gestural input method allowed them to interact with the system easily, without knowing about GIS concepts and database queries. The results indicate a higher learnability in the usage of multimodal systems concerning GIS while the system was generally accepted by the participants.

Gold et al. expand the proprietary 3D GIS GeoScene3D with functionality of a collision detection algorithm to support marine traffic monitoring. In addition to marine features, marine objects were integrated into the system, e.g. depth contours in coastal areas, navigational lights, anchorage areas, precautionary areas and even ship wrecks [6. The interaction with the 3D GIS was constrained through the use of a wheel mouse. Gold et al. describe the interaction as quickly learnable by young and old users likewise but also mention constrains that occurred through the restrictions of mouse input: To deal with different affordances in spatial input Gold et al. implemented a manipulator which enabled the user between different modes to map the same mouse gesture to different types of input depending on the intention GeoScene3D. With Kinoogle Boulos et al. created a gestural interface to operate Google Earth via the Microsoft Kinect [1]. Kinoogle is a composition of middle-ware which maps gestural input captured by 
the Kinect and processed by OpenNI and NITE4 to the standard interaction set of Google Earth. OpenNI5 features processing of the image and depth stream of the Kinect while NITE provides extraction of human body motion and allows the definition of full body gestures. Boulos et al. describe full-body gestural input as to be quite exertive but as a great potential to improve user experience e.g., when interacting with large and stereoscopic displays.

Bruder et al. 3] conducted an evaluation on mid-air selection performance of virtual objects in a stereoscopic virtual reality table environment using three different selection techniques for stereoscopic environments with non-GI systems.

1. Direct input, where the user uses the index finger's tip to interact with the virtual object, bears accommodation problems for the human eye as the focus distance among the screen's surface and the finger tip are divergent. The accommodation of the index finger's tip makes the virtual object appear blurred to the user which breaks the stereoscopic effect as accommodation is an oculomotoric depth criterion for visual depth perception [15].

2. Distant input, where the user's index finger tip's position is mapped to a virtual cursor which is set off 10 centimeters to the index finger tip. While the index finger is still resided in the stereoscopic environment and blurred to the users eye a focus loss is avoided by mapping the interaction space into the virtual environment.

3. Distant input with a virtual hand, which is operated by the user in a distance to the user's real hand of 10 centimeters, similar to the second method. The user's real hand remains blurred in the stereo-scopic environment but is not focused during interaction tasks.

The results by Bruder et al. show a better performance in interaction tasks on using distant input and distant input with a virtual hand on doing a selection test based on Fitt's Law 4 with a slightly increased performance on distance pointing using a cursor.

\section{Development of Interaction Concept}

The development of an interaction concept for a GIS in maritime context requires expert knowledge in terms of scenario planning, task descriptions for future planning of use cases as well as special cases concerning the conception of a man-machine interface in on- or offshore use. For this purpose the ATLAS ELEKTRONIK GmbH provided experts from the maritime field that currently are involved into user interface development for onboard systems, with some of them having a seafaring background. The ATLAS ELEKTRONIK GmbH is a naval and marine electronics business company domiciled in Bremen, Germany, specialized on integrated maritime electronic systems and sonar sensors.

\footnotetext{
${ }^{4}$ http://www.openni.org/files/nite/

${ }^{5}$ http://www . openni.org/
} 


\subsection{Guided Expert Interview}

A group of five maritime experts of the ATLAS ELEKTRONIK GmbH were surveyed via guided expert interviews [11, [12 to further investigate the field. All participating experts had basic experience with touch-based interaction in 2D GIS by the time of the guided interview which was divided into three categories: Stereoscopic Visualization, Interaction and Scenario-based Application.

Categories one and two analyzed the experts state of knowledge concerning each topic while category three reconsidered the first two categories in the context of possible scenario-based applications. Each category was arranged as a set of standardized questions concerning to each topic. The interview guideline's consistency was analyzed during a pretest with an extra individual participant. Each interview was scheduled to 45 minutes and the interviewer annotated the statements of the interviewees during the interview since none of the interviewees agreed on live audio recordings.

\subsection{Interview Analysis}

The analysis of results of the guided interviews illustrate possible fields of application for virtual reality GIS software with gesture-based input. The combination of a 3D visualization with a natural user interface was rated as beneficial by the experts for three maritime scenarios:

\section{Harbor Maintenance and Surveillance}

Data for harbor maintenance and surveillance usually converges in onshore control rooms where data of security and safety systems is reviewed and analyzed in real-time. Besides the planning and coordination of arriving ships and harbor personnel data of harbor facilities like surveillance cameras and underwater inspection data is reviewed. A 3D virtual reality visualization having interactive access on real-time data of all installed sensors in a harbor offers possibilities of a central data reviewing element and mastering high information density.

2. Offshore Wind Park Facility Surveillance and Maintenance

Offshore wind park facilities deserve special protection due to their high infrastructural relevance. Besides surveillance technology like cameras or radar, underwater sensors come into operation for maintenance tasks like echo sounders or under water cameras. An interactive $3 \mathrm{D}$ visualization can be a supportive tool for maintenance tasks but also implies challenges concerning reliability issues in terms of gesture-based interaction since an interactive system must reliably work aboard even under swelling sea states concerning to the experts which requires extra research.

\section{Offshore Bathymetry and Economic Geology}

Bathymetric maps and sonographic recordings of ocean beds and sea grounds are 3D recordings generated by echo sounders. A 3D representation of recorded data in a virtual environment is beneficial for planning and evaluating the allotment of offshore resources concerning expert opinion. A combination of 
echo sounder data sets with sea map data also allows safer maritime navigation in shallow waters. The use of a gesture-based interaction in swelling sea states also applies for this scenario which requires extra research.

The aforementioned scenarios form the basis for deriving use case descriptions for a planned prototype. The scenarios can be arranged into two general subjects. Scenario 1 and 2 both describe general interaction using a maritime GIS on- and offshore while scenario 3 is more confined by the idea of working on raw data sets for their analysis. For further classification of use case descriptions one of the three elaborated scenarios is selected for future planning and conversion: Harbor Maintenance and Surveillance. Harbors are central elements in maritime infrastructure with a multitude of safety-critical concerns. A quick access to requested data must be guaranteed with a reliable and robust human-computer interaction. With its multitude on actors and sensors the harbor security and maintenance scenario offers diverse potential for the definition of use-cases for gesture-based interaction in GIS. Safety critical issues in this scenario facilitate increased requirements on reliability. The interviews' results and analysis form the basis of the creation of a scenario-based interaction concept for touchless interaction with GIS in maritime context.

\subsection{Basic Operations in Maritime GIS}

Further development of the maritime GIS touchless interaction concept requires a look at GIS functionality in general. Goodchild summarized use case scenarios to possible use case groups concerning the functionality in GIS, each based on its conceptual sophistication [7]. Table[1] shows four of the originally six groups, extended with exemplary tasks including an interaction target which counts as manipulation object in the virtual system. The classification of the functionality into basic and complex indicates the number of repetitive or non-repetitive steps to achieve completion of the task. Basic indicates one step where complex indicates more than one step.

Table 1. Functionality for the maritime GIS application

\begin{tabular}{|c|c|c|c|}
\hline Functionality & Exemplary Task & Target & Classification \\
\hline \hline query and reasoning & change map or data view & virtual camera & basic \\
\hline measurement & measure area or track & virtual object & complex \\
\hline transformation & manipulate or create data & virtual object & complex \\
\hline descriptive summaries & tag and group objects or data & virtual object & complex \\
\hline
\end{tabular}

\subsection{Hardware Setup}

The implementation of the planned prototype is conceptualized as a componentoff-the-shelf system (COTS) consisting of a Leap Motion controller for handgesture tracking, the Microsoft Kinect sensor for upper-body and head-tracking, 
and a 27 inch passive stereoscopic display, operated with an off-the-shelf laptop with dedicated graphics hardware. On the software side the open-source GIS NASA World Wind Java is used which provides all basic functionality of a GIS software and permits efficient expansion of the available source code with a gestural interaction. The system will be provided with an interface that allows the linking of external real-time sensor data for scenario-related experimental purposes.

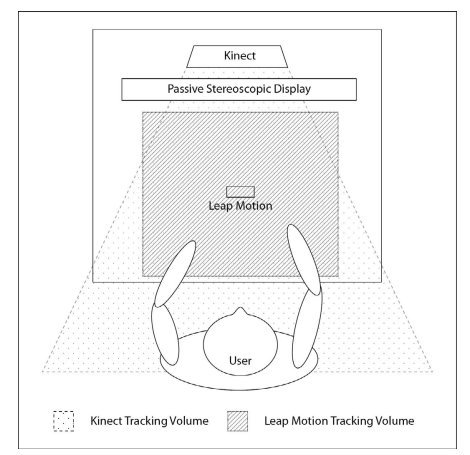

Fig. 1. Workplace as head-tracked stereoscopic environment with gestural interaction

Using both a stereoscopic visualization and coupling of the viewer's head to the virtual camera, enables an immersive virtual reality setting where the user's eyes are not covered as in a setting where virtual reality glasses as e.g. the Oculus Rift 6 are used. This setting allows easy integration of a virtual reality workspace into everyday workspaces [16] as the planned prototype is designated for use in harbor control rooms. Also hand-eye coordination remains unaffected in a stereoscopic head-tracked environment, which additionally allows usage of manual pointing tools for a possible tangible [8] interaction setup for future ideas. Therefore, the leap motion device provides high accuracy on two-hand input and manual tool recognition which can be held in the users tracked hand e.g. as pointing devices.

\subsection{Gestural Interaction Model}

The division of interaction tasks into basic and complex operations suggests an assignment of complex tasks to the user's preferred hand and execution of basic tasks to the user's non-preferred hand [9]. On the basis of the findings of Bruder et al. concerning interaction models and mid-air selection performance in stereoscopic environments [3] the choice on using the Leap Motion controller as gestural input device is consolidated for use in stereoscopic GIS. This affirms

${ }^{6}$ http://www.oculusvr.com/ 
the idea of a gestural input method with two-hand motion in front of a stereoscopic display. Hence, the concept for a distant input method with a virtual visualization of the user's preferred hand forms the general guideline for further elaboration of interaction concepts for the 3D maritime GIS application. While the preferred hand is visualized in the stereoscopic environment for complex task interaction the non-preferred hand remains unvisualized but should be used as input hand for basic tasks simultaneously.

The human hand consists of 16 rigid elements: Three phalanges of each finger plus the palm. The Leap Motion sensor's algorithmic processing in its current state is capable of tracking each finger by providing position and velocity of each finger tip and the position of the palm. These basic capabilities are sufficient to create concepts of differently visualized hand interaction models for later implementation into the maritime GIS as an interactive prototype and later evaluation. Three different visualizations of virtual hand models are presented in the following in general terms.

1. Convex Hand Model. In the convex hand model each finger with their finger tips and the palm of the preferred hand are represented by convex primitives 2. This approach involves potential occlusion of interaction objects which can potentially dissolved by adding different levels of translucency to the convex Hand Model.

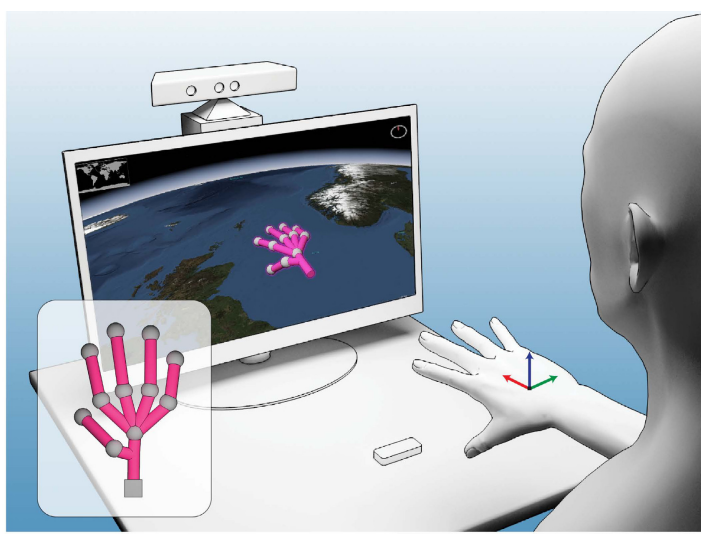

Fig. 2. Interaction model of preferred hand using a convex hand model

2. Kinematic Hand Model. The kinematic hand model is represented by a non-volumetric virtual model of the user's hands functional structure visualized through lines 3 . The lines thickness is planned as to be adjustable (here shown using a thicker line visualization). 


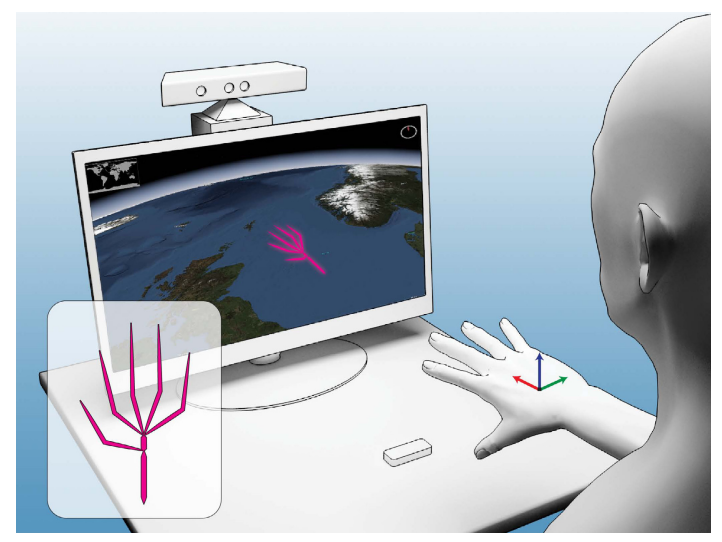

Fig. 3. Interaction model of preferred hand using a kinematic hand model

3. Point Cloud Model. The point cloud model represents the key features of the user's hand by visualizing finger tips and ends, and the palm's mass centre by points 4 . The points' size is planned as to be adjustable.

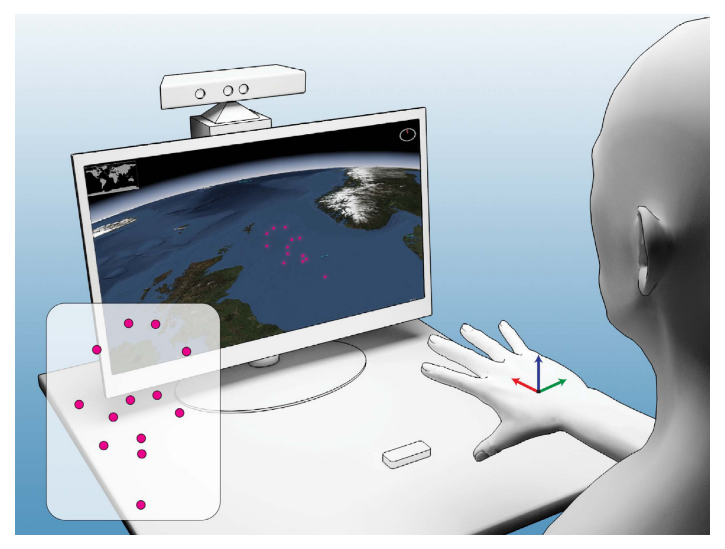

Fig. 4. Interaction model of preferred hand using a point cloud model

\section{Summary and Outlook}

This work elaborated a basic interaction concept for touchless human-computer interaction for geographic information systems in maritime context. Experts from the maritime field were interviewed for the development of possible maritime scenarios and as a basis for the future planning of use cases. The scenario "Harbor Security" has been identified as having a high potential for future conversion since this scenario implies a variety of actors, potential interaction objects 
and different sensors. Additionally, the scenario features particular requirements in terms of reliability as harbors are highly frequented areas where clear view on available system data with rapid cognitive information processing and reaction times are indispensable. Basic GIS operations have been tabled and been purposed as basic and complex interaction tasks for the two-hand touchless interaction concept, in which the user's non-preferred hand operates basic tasks while the users preferred hand operates complex tasks. Different hand models for virtual object interaction have been elaborated and presented. All necessary hardware and software components were arranged for a first stereoscopic GIS prototype. Future steps are the implementation of the elaborated scenario into the stereoscopic GIS environment and the integration of the COTS sensor hardware into the GIS software. The implementation of the presented touchless interaction model will be followed by an evaluation in which the three virtual hand models, presented in this work, will be further investigated.

\section{References}

1. Boulos, M.K., Blanchard, B.J., Walker, C., Montero, J., Tripathy, A., GutierrezOsuna, R., et al.: Web GIS in practice x: A microsoft kinect natural user interface for google earth navigation. International Journal of Health Geographics 10(1), 45 (2011)

2. Bowman, D.A.: 3D user interfaces: Theory and practice. Addison-Wesley, Boston (2005)

3. Bruder, G., Steinicke, F., Strzlinger, W.: Effects of visual conflicts on 3D selection task performance in stereoscopic display environments. In: Proceedings of IEEE Symposium on 3D User Interfaces 3DUI, pp. 115-118. IEEE Press (2013)

4. Fitts, P.M.: The information capacity of the human motor system in controlling the amplitude of movement. Journal of Experimental Psychology 47(6), 381 (1954)

5. Fuhrmann, S., MacEachren, A., Dou, J., Wang, K., Cox, A.: Gesture and speechbased maps to support use of GIS for crisis management: A user study. AutoCarto 2005 (2005)

6. Gold, C., Chau, M., Dzieszko, M., Goralski, R.: 3D geographic visualization: The marine GIS. In: Developments in Spatial Data Handling, pp. 17-28. Springer, Heidelberg (2005)

7. Michael, F.: Goodchild. The use cases of digital earth. International Journal of Digital Earth 1(1), 31-42 (2008)

8. Ishii, H.: The tangible user interface and its evolution. Commun. ACM 51(6), 32-36 (2008)

9. Kabbash, P., MacKenzie, I.S., Buxton, W.: Human performance using computer input devices in the preferred and non-preferred hands. In: Proceedings of the INTERACT 1993 and CHI 1993 Conference on Human Factors in Computing Systems, pp. 474-481. ACM (1993)

10. Khoshelham, K., Elberink, S.O.: Accuracy and resolution of kinect depth data for indoor mapping applications. Sensors 12(2), 1437-1454 (2012)

11. Küsters, I.: Narrative Interviews: Grundlagen und Anwendungen. Springer, Heidelberg (2009)

12. H.O. Mayer.: Interview und schriftliche Befragung: Grundlagen und Methoden empirischer Sozialforschung. Oldenbourg Verlag (2012) 
13. Petit, M., Ray, C., Claramunt, C.: A contextual approach for the development of GIS: application to maritime navigation. In: Carswell, J.D., Tezuka, T. (eds.) W2GIS 2006. LNCS, vol. 4295, pp. 158-169. Springer, Heidelberg (2006)

14. Rauschert, I., Sharma, R., Fuhrmann, S., Brewer, I., MacEachren, A.: Approaching a new multimodal gis-interface. In: Proceeding of the 2nd International Conference on GIS GIScience, CO, USA (2002)

15. Schlick, C., Luczak, H., Bruder, R.: Arbeitswissenschaft. Springer, Heidelberg (2010)

16. Ware, C., Arthur, K., Booth, K.S.: Fish tank virtual reality. In: Proceedings of the INTERACT 1993 and CHI 1993 Conference on Human Factors in Computing Systems, CHI 1993, New York, NY, USA, pp. 37-42. ACM (1993)

17. Weichert, F., Bachmann, D., Rudak, B., Fisseler, D.: Analysis of the accuracy and robustness of the leap motion controller. Sensors 13(5), 6380-6393 (2013)

18. Yu, L.-J., Sun, D.-F., Peng, Z.-R., Zhang, J.: A hybrid system of expanding 2D GIS into 3D space. Cartography and Geographic Information Science 39(3), 140-153 (2012) 